\title{
Medicinal plant phytochemicals and their inhibitory activities against pancreatic lipase: molecular docking combined with molecular dynamics simulation approach
}

\author{
Bilal Ahmed, Usman Ali Ashfaq \& Muhammad Usman Mirza
}

To cite this article: Bilal Ahmed, Usman Ali Ashfaq \& Muhammad Usman Mirza (2017): Medicinal plant phytochemicals and their inhibitory activities against pancreatic lipase: molecular docking combined with molecular dynamics simulation approach, Natural Product Research, DOI: 10.1080/14786419.2017.1320786

To link to this article: http://dx.doi.org/10.1080/14786419.2017.1320786

+ View supplementary material ๔ك

曲 Published online: 26 Apr 2017.

Submit your article to this journal $₫$

Џll Article views: 116

Q View related articles $\sqsubset$

View Crossmark data $־$ 


\title{
Medicinal plant phytochemicals and their inhibitory activities against pancreatic lipase: molecular docking combined with molecular dynamics simulation approach
}

\section{Bilal Ahmed ${ }^{a}$, Usman Ali Ashfaq a and Muhammad Usman Mirza ${ }^{b}$}

a Department of Bioinformatics \& Biotechnology, Government College University Faisalabad (GCUF), Faisalabad, Pakistan; ' Department of Pharmaceutical and Pharmacological Sciences, Rega Institute for Medical Research, Medicinal Chemistry, University of Leuven, Leuven, Belgium

\begin{abstract}
Obesity is the worst health risk worldwide, which is linked to a number of diseases. Pancreatic lipase is considered as an affective cause of obesity and can be a major target for controlling the obesity. The present study was designed to find out best phytochemicals against pancreatic lipase through molecular docking combined with molecular dynamics (MD) simulation. For this purpose, a total of 3770 phytochemicals were docked against pancreatic lipase and ranked them on the basis of binding affinity. Finally, 10 molecules (Kushenol K, Rosmarinic acid, Reserpic acid, Munjistin, Leachianone G, Cephamycin C, Arctigenin, 3-O-acetylpadmatin, Geniposide and Obtusin) were selected that showed strong bonding with the pancreatic lipase. MD simulations were performed on top five compounds using AMBER16. The simulated complexes revealed stability and ligands remained inside the binding pocket. This study concluded that these finalised molecules can be used as drug candidate to control obesity.
\end{abstract}
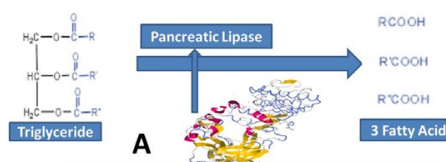

$\mathrm{RCOOH}$
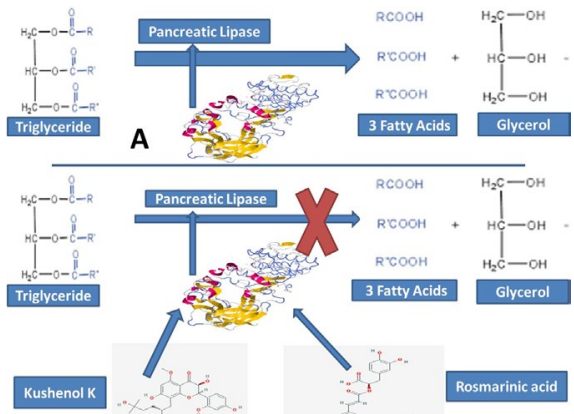

B

CONTACT Usman Ali Ashfaq usmancemb@gmail.com

(4) Supplemental data for this article can be accessed at https://doi.org/10.1080/14786419.2017.1320786. 


\section{Introduction}

Obesity is a term which is described as the accumulation of immense fat in the human body. Obesity is the worst health risk worldwide (Sukhdev \& Singh 2013). It is commonly described by Body mass index (BMI). BMI is the ratio of object's weight to height (Locke et al. 2015). It is a measure commonly used to assess the obesity, if the value of BMI in adults is more than $30 \mathrm{~kg} / \mathrm{m}^{2}$, it means the object is obese. Obesity is a commonly increasing problem around the world. The frequency of obesity is $15-20 \%$ and $10-15 \%$ in women and men, respectively (Seidell 2000; Locke et al. 2015). Obesity is a health risk which is attached with a number of diseases like dyslipidemia, sleep apnoea (Sukhdev \& Singh 2013), diabetes mellitus (Archana et al. 2010), hypertension (Vaněčková et al. 2014) and heart disease (Bastien et al. 2014; Lavie et al. 2014; Nakamura et al. 2014). According to the WHO survey there were 1.9 billion overweighted, 41 million children under the age of 5 and 600 million adults were obese in 2014 . Main cause of obesity is imbalance between energy intake and use (Lavie et al. 2014). Less physical activity, overeating and intake of food which is rich in dietary fat are the main reasons for obesity.

Pancreatic lipase is an enzyme which is produced in pancreas and secreted into the intestine. Pancreatic lipase converts triglycerides into fatty acids and therefore it is considered as an affective cause of obesity (Mukherjee \& Sengupta 2013). Pancreatic lipase can be a major target for controlling the obesity because it has a vital role in increasing the body weight.

Thousands of plants and their extracts are used as a source of medicine. Number of phytochemicals are characterised and separated from different plant sources such as saponins, flavonoids, flavonols and alkaloids. Phytochemicals may act as good anti-obesetic agents by minimising the absorption of lipids and energy intake from food and by increasing the use of energy by degrading the fat which is stored in the human body (Sukhdev \& Singh 2013; Mukherjee et al. 2015; Masmoudi-Allouche et al. 2016). Therefore, this study is conducted to increase the range of pancreatic lipase inhibitors which has fewer side effects as compared to 'Orlistat'.

\section{Results and discussion}

Pancreatic lipase structure was taken from PDB and optimised with the help of MOE. Pancreatic lipase was docked against a library of ligand molecules. MOE sorted the result by docking score and gave the possible confirmations for each ligand molecule. Top confirmations of each ligand molecule were further analysed and were used to check the interactions with the target protein. Ten molecules were finalised based on their binding affinities and drug-likeness screening. Pubchem_ID, docking score, root mean square deviation values and residues of target molecule which interact with the ligand molecule are given in (Table 1)). Further, 20 ns MD simulations were performed for the five top-ranked complexes to check the stability of protein's back bone and to understand the interacting behaviour of the ligand after simulation.

\subsection{Molecular docking studies}

Orlistat is a drug which acts as a lipase inhibitor and mainly used as an anti-obesitic agent. It plays a major role in controlling the obesity and was used as a reference drug in the current study Molecular weight of kushinol K is $472.534 \mathrm{~g} / \mathrm{mol}$. It contains five hydrogen bond donors 
Table 1. Docking results of top 10 molecules and control drug are given here. Ser 152 is highlighted in red which indicates that all the selected molecules interact with it just like Orlistat.

\begin{tabular}{lcccl}
\hline Sr_NO & Pubchem_ID & Docking score & RMSD refine & Interacting residues \\
\hline 1 & $44,428,630$ & -13.7835 & 1.1342 & Ser152, Arg256, Asp79 \\
2 & $5,281,792$ & -13.5563 & 1.0924 & Ser152, Tyr114, Phe215, His263 \\
3 & 65,747 & -13.2923 & 1.1394 & Ser152 \\
4 & 160,476 & -13.2506 & 1.2199 & Ser152, Phe215, Tyr114, His263 \\
5 & $5,275,227$ & -13.1246 & 1.4942 & Ser152, Asp79, Arg256, His151, His263 \\
6 & 155,380 & -12.8606 & 1.8984 & Ser152, Arg256, Phe77, Phe215, His263 \\
7 & 107,848 & -12.5235 & 1.2337 & Ser152, Phe77, Phe215, His151, His263 \\
8 & 441,084 & -12.3431 & 2.0383 & Ser152, Phe215, Ile78 \\
9 & 64,981 & -12.1382 & 1.3025 & Ser152, His263 \\
10 & $10,406,203$ & -12.0290 & 0.9371 & Ser152, His263 \\
11 & Orlistat(Ref) & -10.2014 & 3.5768 & Ser152 \\
\hline
\end{tabular}

and eight hydrogen bond acceptors. It showed strong bonding and less docking score $(-13.78 \mathrm{kcal} / \mathrm{mol})$ as compared to the reference drug $(-10.2014 \mathrm{kcal} / \mathrm{mol})$. It also showed strong interactions with the different residues of target molecule i.e. Ser152, Arg256 and Asp79 within the same pocket, where reference drug bound as shown in (Figure 1). All other selected molecules shown in Table 1 had significant binding potential as well as less docking score, from -13.55 to $-12.02 \mathrm{kcal} / \mathrm{mol}$, as compared to the reference drug. Interactions of selected molecules are shown in (Figure 1) and strong binding modes of selected phytochemicals with the target molecule are given in (Figure 2).

In a recent study, it has been reported that chlorophyll a, Isofucosterol and Saringosterol, found in Sargassum thunbergii, act as potential inhibitors of pancreatic lipase (Kim et al. 2014). Docking score and interaction study of the reported compounds have been compared with the compound proposed in present study which indicated that, compounds reported in current study show a strong interaction with the pancreatic lipase as compared to the already reported molecules.

\subsection{Drug-like properties}

Drug scan was performed to assess the drug-like properties of all the finalised phytochemicals. Lipinski's Rule of Five was used as a standard. This rule explains the different properties of a drug molecule as well as its interactions metabolism within the human body as well as its excretion. Drug-like properties of all the finalised molecules, as well as reference drug, are given in (Table S1). All the selected molecules of this study followed the rule and showed all those properties which a drug should contain.

\subsection{Molecular dynamics simulation studies}

For the current study, only the top-ranked compounds that predicted binding affinities lower than $-13 \mathrm{kcal} / \mathrm{mol}$ were taken in consideration for $20 \mathrm{~ns}$ all-atom backbone simulation. These include; Kushenol K, Rosmarinic acid, Reserpic acid, Munjistin and Leachianone G. All these compounds were found to remain inside the binding pocket throughout MD simulation. For all complexes, the computed root-mean-square-deviation (RMSD) using allbackbone atoms on initial minimised docked complex were found to be less than $1.5 \AA, 2.5 \AA, 2.25 \AA$, $1.5 \AA ̊$ and $2 \AA$ against Kushenol K, Rosmarinic acid, Reserpic acid, Munjistin and Leachianone 


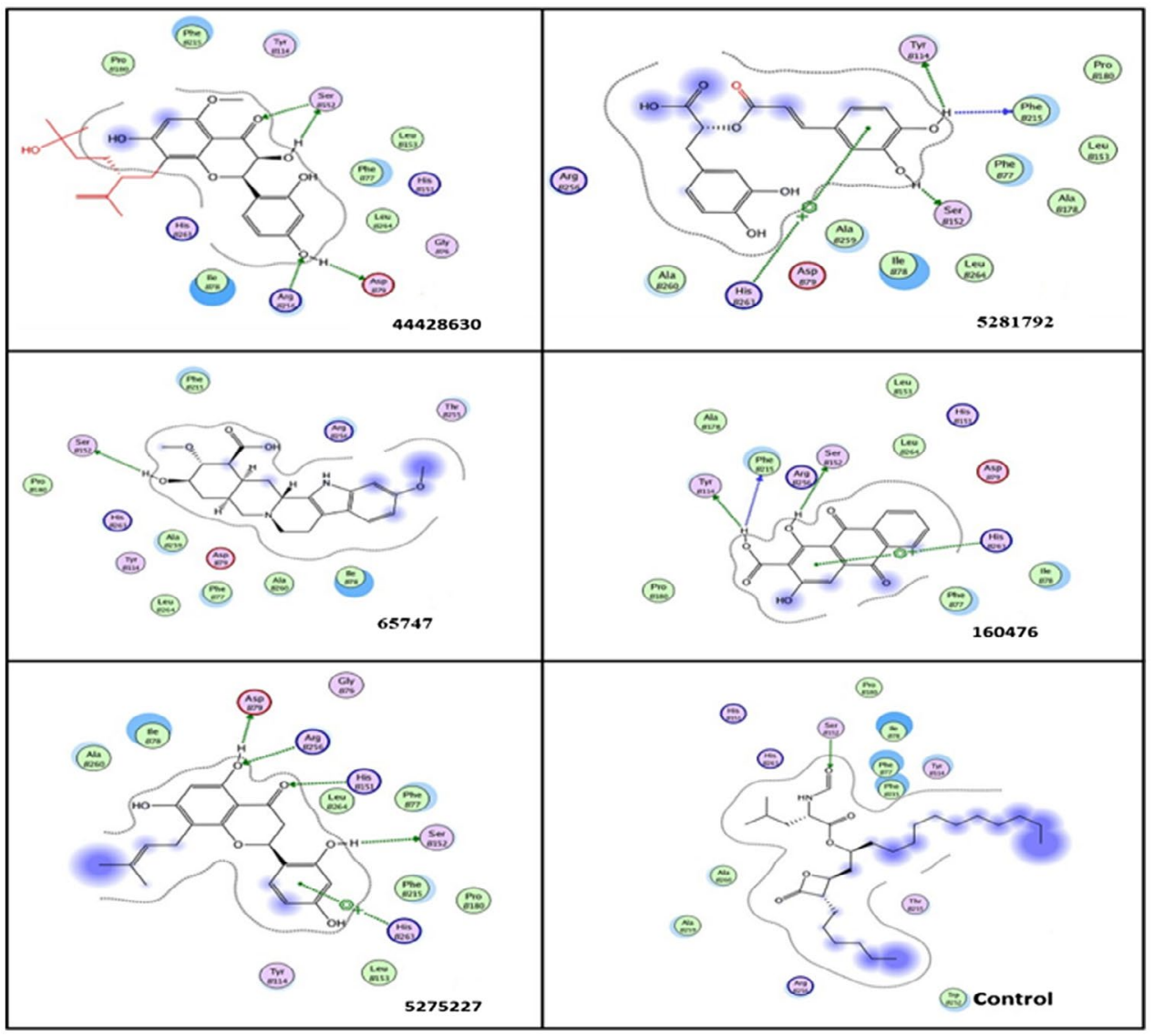

Figure 1. Interactions of first five finalised molecules and control drug with pancreatic lipase are shown in this figure. Interacting residues of receptor with ligand molecules are shown with the help of arrows. Green arrows indicating the side chain acceptors and donors while blue arrows representing the backbone acceptors and donors.

G, respectively (Figure S1). Less deviation from the initial structure showed that the simulated system was well equilibrated. Also, there were less than $0.5 \AA$ deviations with all the five compounds which were in consensus with the initial conformation and these ligands remained inside the binding pocket throughout the 20 ns simulation. While, Kushenol K, Munijistin and Leachianone complexed with pancreatic lipase were found to be more stable as the RMSD of protein's backbone remained to converge between 1-1.5, $0.7-1.4 \AA$ and $1.5-2 \AA$, respectively. To analyse per residue fluctuations, average root-mean-square-fluctuations (RMSF) for each residue throughout simulations are displayed in Figure S2. It can be seen that no big fluctuations are reported, except few with no greater than $2.5 \AA$ because of the presence of flexible loops in that particular region of pancreatic lipase. Importantly, the binding site residues (Phe77 - Ser152) only fluctuated less than $0.75 \AA ̊$ i.e. Ser 152, which was key residue in interaction with all ligands. Apparently to Rosamarinic acid and Resperpic complexes which fluctuated prominently (in orange and red) based on their RMSD deviation plots, Kushenol K (black), Munjistin (Indigo)and Leachianone (Cyan) showed fewer fluctuations. 

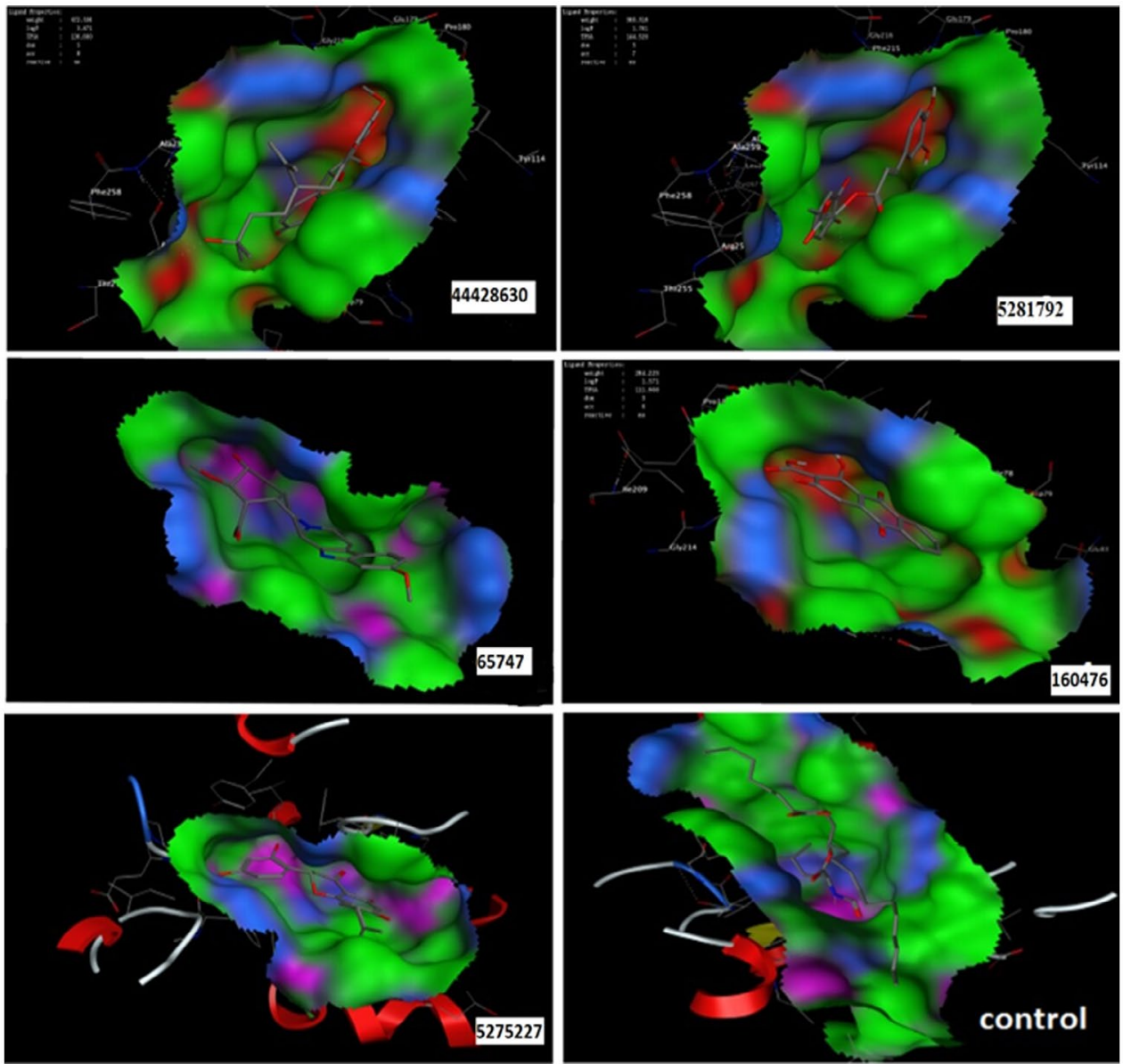

Figure 2. Binding modes of top five finalised molecules and control drug within the pocket of pancreatic lipase. Green surface representing the binding pocket of the receptor, while phytochemicals are shown in ball and stick format.

In summary, our findings reported that Kushenol K with few other compounds showed strong hydrogen bonding and hydrophobic interactions as compared to the reference drug and possessed necessary drug-likeness properties. Furthermore, pancreatic lipase complexed with top compounds remained stable throughout 20 ns MD simulations. Present findings will have a decisive role whether these compounds have the potential to be developed into an effective oral therapeutic inhibitors against pancreatic lipase to control obesity after testing through in vitro and in vivo experiments.

\section{Experimental}

Docking was performed between the target enzyme known as pancreatic lipase and a library of 3770 bioactive compounds with the help of MOE. Top-ranked complexes were then used to perform MD simulation to check the overall stability of binding pose and protein's backbone. 


\subsection{Tools and databases used}

Following tools and databases were used in this present study

Molecular operating Environment (MOE, Roy \& Luck 2007), Protein Data Bank (PDB, Berman et al. 2000), Pubchem (Wang et al. 2009) and AMBER16 for MD simulations (Maier et al. 2015)

\subsection{Preparation of target and ligand molecules}

PDB file of pancreatic lipase was downloaded from the PDB (PBD ID: 1LPA). Co-crystallised ligand molecules, metal atoms and water molecules were removed with the help of MOE. Standard protocol of MOE was used to minimise energy and to set the protonation states. A total of 3770 bioactive molecules belong to the different classes of phytochemicals were taken from the Pubchem. All these molecules were stored in a single database after performing energy minimisation.

\subsection{Molecular docking}

Binding pockets were found with the help of site finder tool of MOE. Before virtual screening, a simple redocking procedure of co-crystallised ligand on the selected protein to check the reliability of scoring function of docking algorithm used. Top conformations were selected on the basis of energy and docking score for further analysis.

\subsection{Drug scan}

After the step of molecular docking, drug-like properties of ligand molecules were checked using the drug scan tool to ensure that the finalised molecules follow the Lipinski's Rule of Five.

\subsection{Molecular dynamics simulation}

MD simulations of 20 ns were performed to check the pancreatic lipase backbone stability with top ranked selected ligands using AMBER 16.0 software package (Case et al. 2014). Amber ff14SB force fields (Maier et al. 2015) were used to describe proteins counter ions and water. Antechamber programme was employed to generate force field parameters for ligands using AMBER force field (GAFF) using the Antechamber programme. The tleap module was used to produce all essential parameters by considering residues at their default protonation states at neutral $\mathrm{pH}$ value. Prior to energy minimisation, all complexes were neutralised by adding $\mathrm{Na}+$ ions around the complex at different locations and centred in a truncated octahedron box with a distance of $10 \AA$ from the edge of the box, and solvated using TIP3P water molecules. The particle mesh Ewald method with a non-bounded cut-off of $10 \AA$ was used to set the periodic boundary conditions. Integration step at $2 \mathrm{fs}$ was set and the SHAKE was employed to constrain $\mathrm{X}-\mathrm{H}$ bond lengths involving hydrogen bonds. The system and counter ions, water simulated underwent minimisation using 5000 steps of steepest descent method followed by conjugate gradient method. Then system was gradually heated from 0 to $300 \mathrm{~K}$ with $50 \mathrm{ps}$ and maintained at $300 \mathrm{~K}$ with restraints on the complex. After heating, the system was equilibrated for 500 ps and finally, a 20 ns of a relaxed MD production run was performed 
using constant pressure $(p=1.0 \mathrm{~atm})$ and temperature $(T=300 \mathrm{~K})$. Coordinate trajectories were collected after every 2 ps for the whole production run and CPPTRAJ modules of AMBER 16 were implied for trajectories analyses (Roe \& Cheatham 2013).

\section{Acknowledgments}

The authors would like to acknowledge Department of Bioinformatics and Biotechnology, Government College University, Faisalabad, 38000, Pakistan.

\section{Disclosure statement}

No potential conflict of interest was reported by the authors.

\section{References}

Archana P, Sathishkumar N, Bharathi N. 2010. In silico docking analysis of curcumin-an inhibitor for obesity. Int J Pharm Bio Sci 1:224-235.

Bastien M, Poirier P, Lemieux I, Després J-P. 2014. Overview of epidemiology and contribution of obesity to cardiovascular disease. Prog Cardiovasc Dis. 56:369-381.

Berman HM, Westbrook J, Feng Z, Gilliland G, Bhat TN, Weissig H, Shindyalov IN, Bourne PE. 2000. The protein data bank. Nucleic Acids Res. 28:235-242.

Case DA, Babin V, Berryman VJT, Betz RM, Cai Q, Cerutti DS, Cheatham TE, Darden TA, Duke RE, Gohlke $\mathrm{H}$, et al. 2014. Amber 14. San Francisco: University of California.

Kim KBWR, Kim MJ, Ahn DH. 2014. Lipase inhibitory activity of chlorophyll a, isofucosterol and saringosterol isolated from chloroform fraction of Sargassum thunbergii. Nat Prod Res. 28:1310-1312.

Lavie CJ, McAuley PA, Church TS, Milani RV, Blair SN. 2014. Obesity and cardiovascular diseases: implications regarding fitness, fatness, and severity in the obesity paradox. J Am Coll Cardiol. 63:1345-1354.

Locke AE, Kahali B, Berndt SI, Justice AE, Pers TH, Day FR, Powell C, Vedantam S, Buchkovich ML, Yang J. 2015. Genetic studies of body mass index yield new insights for obesity biology. Nature. 518:197-206.

Maier JA, Martinez C, Kasavajhala K, Wickstrom L, Hauser KE, Simmerling C. 2015. ff14SB: improving the accuracy of protein side chain and backbone parameters from ff99SB. J Chem Theory Computation. 11:3696-3713.

Masmoudi-Allouche F, Touati S, Mnafgui K, Gharsallah N, El Feki A, Allouche N. 2016. Phytochemical profile, antioxidant, antibacterial, antidiabetic and anti-obesity activities of fruits and pits from date palm (Phoenix dactylifera L.) grown in south of Tunisia. J Pharmacogn Phytochem. 5:15-22.

Mukherjee A, Sengupta S. 2013. Indian medicinal plants known to contain intestinal glucosidase inhibitors also inhibit pancreatic lipase activity-An ideal situation for obesity control by herbal drugs. IJBT. 12:32-39.

Mukherjee A, Mukherjee S, Biswas J, Roy M. 2015. Phytochemicals in obesity control. Int J Curr Microbiol App Sci. 4:558-567.

Nakamura K, Fuster JJ, Walsh K. 2014. Adipokines: a link between obesity and cardiovascular disease. Int J Cardiol. 63:250-259.

Roe DR, Cheatham TE III. 2013. PTRAJ and CPPTRAJ: software for processing and analysis of molecular dynamics trajectory data. Journal of Chemical Theory and Computation. 9:3084-3095.

Roy U, Luck LA. 2007. Molecular modeling of estrogen receptor using molecular operating environment. Biochem Mol Biol Edu. 35:238-243.

Seidell JC. 2000. Obesity, insulin resistance and diabetes-A worldwide epidemic. Br J Nutr. 83:S5-S8.

Sukhdev S, Singh K. 2013. Therapeutic role of phytomedicines on obesity: importance of herbal pancreatic lipase inhibitors. Int Res J Med Sci. 1:15-26.

Vaněčková l, Maletínská L, Behuliak M, Nagelová V, Zicha J, Kuneš J. 2014. Obesity-related hypertension: possible pathophysiological mechanisms. J Endocrinol. 223:R63-R78.

Wang Y, Xiao J, Suzek TO, Zhang J, Wang J, Bryant SH. 2009. PubChem: a public information system for analyzing bioactivities of small molecules. Nucleic Acids Res. 37:W623-W633. 